$16,12,03$

\title{
Атомная структура и энергия когезии кластеров ZnSe и CdSe
}

\author{
(С) Л.И. Овсянникова \\ Институт проблем материаловедения им. И.Н. Францевича НАН Украины, \\ Киев, Украина \\ E-mail: avilon57@ukr.net
}

(Поступила в Редакцию 29 ноября 2018 г.

В окончательной редакции 29 ноября 2018 г.

Принята к публикации 5 декабря 2018 г.)

\begin{abstract}
Проведены первопринципные расчеты атомной и электронной структуры фуллереноподобных кластеров $\mathrm{Zn}_{n} \mathrm{Se}_{n}$ и $\mathrm{Cd}_{n} \mathrm{Se}_{n}$ для $n=12,36,48,60$. Впервые построена модель двухслойных фуллереноподобных кластеров $(\mathrm{ZnSe})_{60}$ и $(\mathrm{CdSe})_{60}$ со смешанными $s p^{2} / s p^{3}$-связями. Ab initio расчеты проводились в рамках теории функционала электронной плотности и гибридного функционала B3LYP. Проведена оценка стабильности и ширины энергетической щели кластеров в зависимости от количества атомов в кластере и его геометрии. Показано, что в процессе релаксации двухслойных атомных кластеров $(\mathrm{ZnSe})_{60}$ и $(\mathrm{CdSe})_{60}$ со смешанными $s p^{2} / s p^{3}$-связями размером $1.7-1.8 \mathrm{~nm}$ происходит отщепление наружноого слоя.
\end{abstract}

DOI: 10.21883/FTT.2019.04.47431.331

\section{1. Введение}

Полупроводники $\mathrm{A}^{2} \mathrm{~B}^{6}$ привлекают большой интерес в связи с их широким применением в оптоэлектронных устройствах для детектирования и стимуляции излучения в инфракрасной и видимой областях спектра. В частности, селенид цинка $-\mathrm{ZnSe}-$ является ключевым материалом для изготовления высокомощных лазеров, инфракрасных окон, линз и призм вследствие очень низкого коэффициента поглощения в интервале длин волн $0.63-18 \mu \mathrm{m}$. Прогресс в конструировании светоизлучающих диодов на основе $\mathrm{ZnSe}$ для зелено-голубой области спектра возродил интерес к фундаментальным свойствам и экспериментальным и теоретическим исследованиям атомных, геометрических и электронных свойств этого материала.

Наряду с твердотельными широкое применение находят полупроводниковые нанокристаллы - квантовые точки (КТ) на основе прямозонных полупроводников $\mathrm{A}^{2} \mathrm{~B}^{6}$ (ZnSe, CdSe, $\mathrm{CdS}$ и т.д.) [1]. Нанокристаллы практически не подвержены деградации по сравнению с органическими люменисцентными материалами и имеют высокий квантовый выход люминесценции. В последнее время можно синтезировать нанокристаллы (КТ) размерами несколько нанометров в диаметре, состоящие из $10^{3}-10^{5}$ атомов. Такие КТ характеризуются дискретным энергетическим спектром, подобным атомному, шириной запрещенной зоны и энергией люменисценции, меняющимися с их размерами. При использовании масштабируемой процедуры синтеза на основе одного и того же материала могут быть получены нанокристаллы с различными спектрами излучения, перекрывающими всю видимую область спектра, поскольку эмиссионные свойства нанокристаллов в первую очередь определяются эффектом размерного квантования [2].

Квантовые точки могут существовать как в виде ядра, так и в виде гетероструктур типа ядро-оболочка, когда флуоресцирующие ядра КТ покрывают слоем более широкозонного полупроводника со схожими структурой и составом, при этом пассивируются поверхностные дефектные состояния ядер КТ. Были синтезированы $\mathrm{CdSe} / \mathrm{nCdS}$ КТ с диаметром CdSe ядра КТ 3.03 nm [3]. $\mathrm{B}$ [4] сообщается о исследованиии пленок $\mathrm{CdSe}, \mathrm{CdTe}$, в которых наночастицы представляют собой почти плотноупакованные сферы диаметром 3-4 nm.

Возможные варианты геометрии КТ не ограничиваются лишь сферическими наночастицами, и в последнее время весьма широкий интерес вызывают полупроводниковые нанокристаллы планарной геометрии (2D-нанокристаллы, нанопластины, нанодиски, наносвитки) [5]. Замечено, что при сворачивании нанопластин $\mathrm{CdSe}$ в структуры винтовой формы происходит красный сдвиг максимума их фотолюминесценции, обусловленный возникновением внутренних полей, связанных с механическими напряжениями при скручивании [6].

Хотя распространенная система для квантовых точек - халькогениды кадмия (CdS, CdSe), но они содержат токсичные соединения. К системам с такими же характеристиками и не содержащих токсичных элементов относится широкозонный $\mathrm{ZnSe}$, который излучает в фиолетовой области. Для управления диапазоном излучения квантовых точек их легируют $3 d$-металлами переходных групп (Mn, Fe, Co, Ni, Cu). Поэтому свойства квантовых точек $\mathrm{ZnSe}, \mathrm{CdSe}$ и кластеров размером в десятки и сотни атомов на основе этих материалов представляют огромный интерес и являются объектом компьютерного моделирования [7,8].

Целью настоящей работы является исследование средствами компьютерного материаловедения структурных, электронных и когезионных свойств изолированных фуллереноподобных кластеров на основе прямозонных полупроводников $\mathrm{A}^{2} \mathrm{~B}^{6}: \mathrm{ZnSe}, \mathrm{CdSe}$ в зависимости от их размера, геометрии и выбора базовой модели для дальнейшего исследования свойств этих кластеров при легировании $3 d$-металлами. 


\section{2. Модель и метод исследования}

Для исследования электронных и когезионных свойств изолированных кластеров на основе прямозонных полупроводников $\mathrm{A}^{2} \mathrm{~B}^{6}$ первопринципными квантово-химическими методами мы предлагаем использовать фуллереноподобные (ФП) модели кластеров размером до $2 \mathrm{~nm}$. ФП-модели успешно применялись при исследовании поведения примеси $\mathrm{Cd}$ в $\mathrm{Zn}(\mathrm{Cd}) \mathrm{O}$ сплавах и взаимодействия собственных дефектов с примесью $\mathrm{Al}$ в решетке $\mathrm{ZnO}$ [9-11]. Мы ранее установили, что $\mathrm{A}^{\mathrm{II}} \mathrm{B}^{\mathrm{VI}}$ полупроводниковые соединения проявляют тенденцию формировать фуллереноподобные структуры [12]. В таких структурах, энергия когезии меньше чем для основных твердотельных структур. Также, энергия когезии увеличивается с ростом числа атомов в кластере, таким образом большие кластеры устойчивее, чем более маленькие. Хотя нет полной ясности в наблюдаемой тенденции сокращения ширины запрещенной зоны с увеличением размера кластера, но значение энергии запрещенной зоны для кластеров, зависит не только от размера кластера - на это могут повлиять и другие факторы - такие как геометрия $[13,14]$.

Для определения размерной зависимости энергии когезии кластеров $\mathrm{ZnSe}$, CdSe нами была проведена сравнительная оценка стабильности ФП $(\mathrm{ZnSe})_{n},(\mathrm{CdSe})_{n}$ кластеров $(n=12,36,48,60)$. Эти кластеры в силу их кубического типа симметрии могут служить структурной единицей цеолитоподобных периодических структур $[15,16]$. Кластеры с двумя вложенными слоями онион $Z_{12} \mathrm{Se}_{12} @ \mathrm{Zn}_{48} \mathrm{Se}_{48}$ и сферический $(\mathrm{ZnSe})_{60}$ кластер со смешанным $s p_{3} / s p_{2}$-типом связи и изоэлектронные им $\mathrm{Cd}_{12} \mathrm{Se}_{12} @ \mathrm{Cd}_{48} \mathrm{Se}_{48}$ и $(\mathrm{CdSe})_{60}$ имеют одинаковое число атомов и построены для исследования свойств кластера от его геометрии, а также исследования поведения поверхностного слоя кластеров в процессе их релаксации.

При построении моделей сферических кластеров можно использовать структурные фрагменты известных кристаллических модификаций исследуемых веществ. Например, фрагменты алмаза и лонсдейлита были использованы для построения модели частиц икосаэдрического наноалмаза в $[17,18]$, в которых локальное окружение атомов углерода практически не отличалось от того, что имеет место в алмазе, а в центре модели располагался фуллерен $\mathrm{C}_{20}$. Мы использовали подход, изложенный в $[17,18]$ для построения модели $(\mathrm{ZnSe})_{60}$ и $(\mathrm{CdSe})_{60}$ кластеров со смешанными $s p^{3} / s p^{2}$-связями (рис. 1).

Модели ФП $(\mathrm{ZnSe})_{n}$ кластеров при $n=12,36,48$ представлены на рис. 2 . Кластер $(\mathrm{ZnSe})_{12}$ симметрии $T_{h}$ образован 6 квадратами и 8 гексагонами, где каждый атом цинка имеет окружение из трех атомов селена и наоборот. Особенность кластера $(\mathrm{ZnSe})_{12}$ в том, что он может служить структурной единицей периодических цеолитоподобных структур с кубической решеткой, где все атомы объединены $s p^{3}$-связями $[15,16]$. Кластер $(\mathrm{ZnSe})_{48}$ симметрии $T_{h}$ имеет форму тетраэдра и состоит из 6 квадратов, расположенных по вершинам тетраэдра,

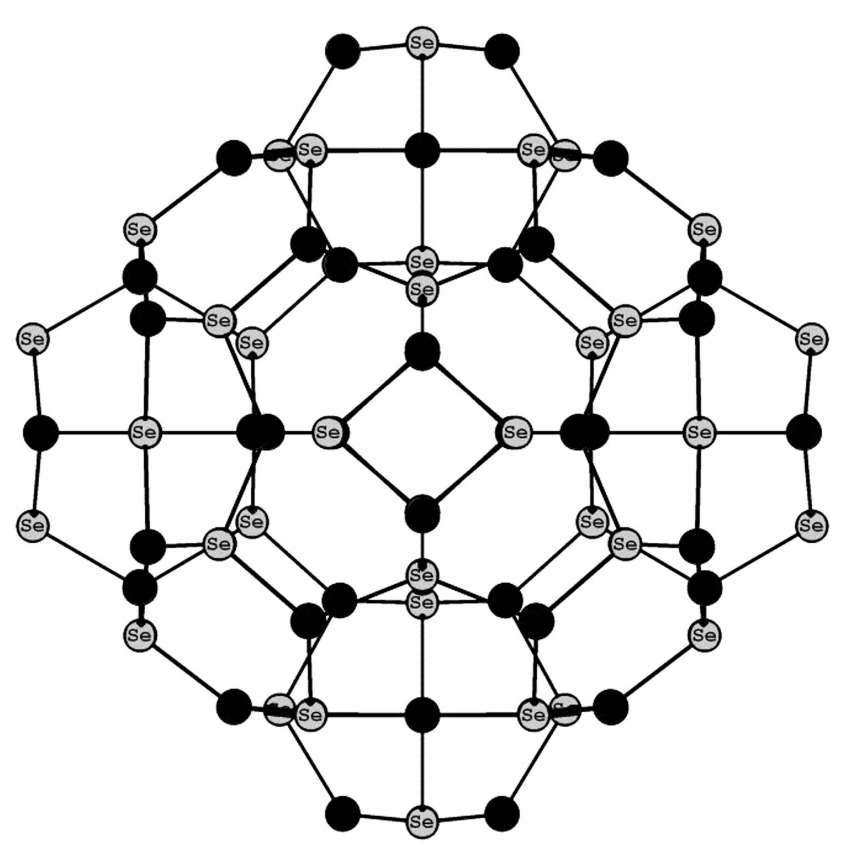

Рис. 1. Исходная модель $(\mathrm{ZnSe})_{60}$ и $(\mathrm{CdSe})_{60}$ кластеров со смешанными $s p^{2} / s p^{3}$-связями, где темные шарики - Me $(\mathrm{Zn}, \mathrm{Cd})$, светлые шарики - Se.

и 40 гексагонов (шестичленных колец), объединеных в 8 плоских фрагментов из 5 гексагонов каждый.

Модели ониона $\mathrm{Zn}_{12} \mathrm{Se}_{12} @ \mathrm{Zn}_{48} \mathrm{Se}_{48}$ и $(\mathrm{ZnSe})_{60}$ кластера со смешанными $s p^{2} / s p^{3}$-связями представлены на рис. 3. Онион построен на основе полого ФП кластера $(\mathrm{ZnSe})_{12}$ вложенного внутрь кластера $(\mathrm{ZnSe})_{48}$ описанных выше. Модель $(\mathrm{ZnSe})_{60}$ кластера построена на основе ядра из кластера $(\mathrm{ZnSe})$ 12. При построении наружного слоя были использованы структурные фрагменты $\mathrm{ZnSe}$ структуры сфалерита. Ядро декорировано структурными фрагментами таким образом, что каждый атом ядра является местом роста следующего сфалеритоподобного слоя. В полученной частице с двухслойной структурой $(\mathrm{ZnSe})_{60}$, из 120 атомов ее составляющих, 48 внутренних атомов объединены $s p^{3}$-связями, а 72 поверхностных атома $s p^{2}$-связями (соотношение числа атомов с $s p^{2} / s p^{3}$-связями составляет 72/48). Предполагается, что данная методика позволит построение модели частицы с многослойной структурой.

Модели $(\mathrm{CdSe})_{n}$ кластеров для $n=12,36,48,60$ построены аналогично вышеописанным $(\mathrm{ZnSe})_{n}$ кластерам при $n=12,36,48,60$ и представлены на рис. 2, 4.

Оптимизированная геометрия, полная энергия, электронная структура, распределение электронной плотности кластеров, расчитаны в рамках метода RHF и теории функционала электроннй плотности DFT c гибридним функционалом B3LYP и базисним набором MINI [19]. Гибридный функционал с точным обменным взаимодействием, рассчитанным методом Хартри-Фока, обеспечивает алгоритм улучшения предсказания многих свойств кластеров, таких как энергия атомизации, длина 


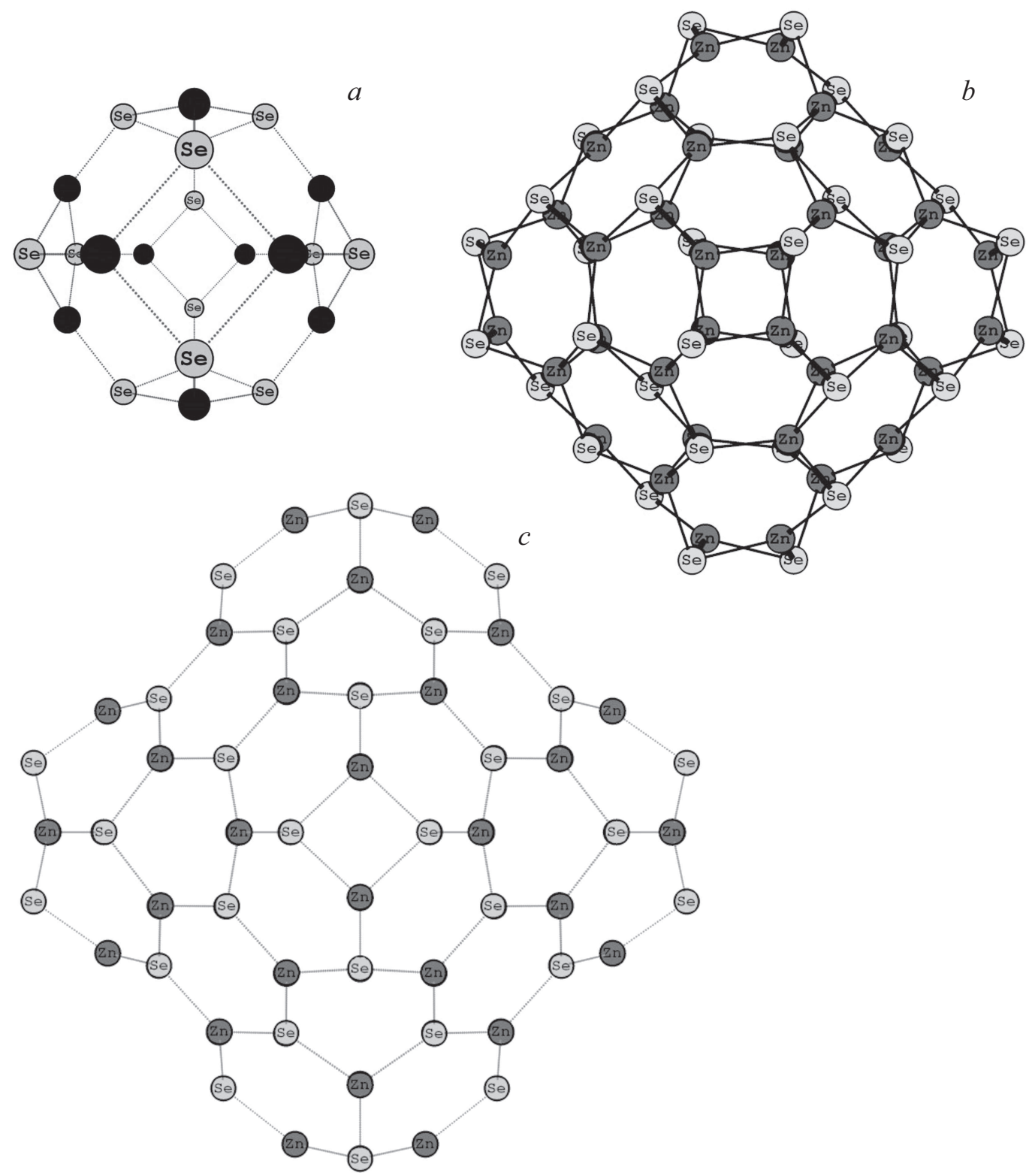

Рис. 2. Оптимизированные геометрии ФП кластеров $\mathrm{Me}_{n} \mathrm{Se}_{n}: n=12(a), n=36(b), n=48(c)$, где темные шарики - $\mathrm{Me}(\mathrm{Zn}, \mathrm{Cd})$, светлые шарики - Se.

химических связей, частоты колебаний. Энергия когезии на пару атомов рассчитывалась как полная энергия кластера за вычетом энергии удаленных в бесконечность составляющих его атомов без учета нулевых колебаний деленная на число атомных пар.

При выполнении расчетов были использованы: комплекс программ GAMESS'09 [20], программы визуализации расчетов ChemCraft и Molekel 5.4 [21].

\section{3. Обсуждение вычислительного эксперимента}

Результатами расчетов явились оптимизированная геометрия и электронная структура исследуемых объек- тов. Результаты расчета электронной структуры, энергии когезии в пересчете на атомную пару $\mathrm{Zn}-\mathrm{Se}$ и $\mathrm{Cd}-\mathrm{Se}$ представлены в табл. 1 и 2 соответственно. Зависимость энергии когезии и ширины енергетической щели в зависимости от размера и геометрии кластера представлены на рис. 5. Вычисления показали, что ширина энергетической щели уменьшается с увеличением размера кластера, а стабильность кластеров (энергия когезии) возрастает с увеличением размера кластера.

Для структур на основе ZnSe наиболее стабильным оказался кластер $\mathrm{Zn}_{48} \mathrm{Se}_{48}$. Онион $\mathrm{Zn}_{12} \mathrm{Se}_{12} @ \mathrm{Zn}_{48} \mathrm{Se}_{48}$, близок к нему, но все же менее стабилен чем $\mathrm{Zn}_{48} \mathrm{Se}_{48}$ (на $0.007 \mathrm{eV}$ на пару $\mathrm{Zn}-\mathrm{Se}$ атомов). Сферический $(\mathrm{ZnSe})_{60}$ оказался метастабилен по отношению к ониону $\mathrm{Zn}_{12} \mathrm{Se}_{12} @ \mathrm{Zn}_{48} \mathrm{Se}_{48}$. Длина связи $\mathrm{Zn}-\mathrm{Se}$ в 6-членном 


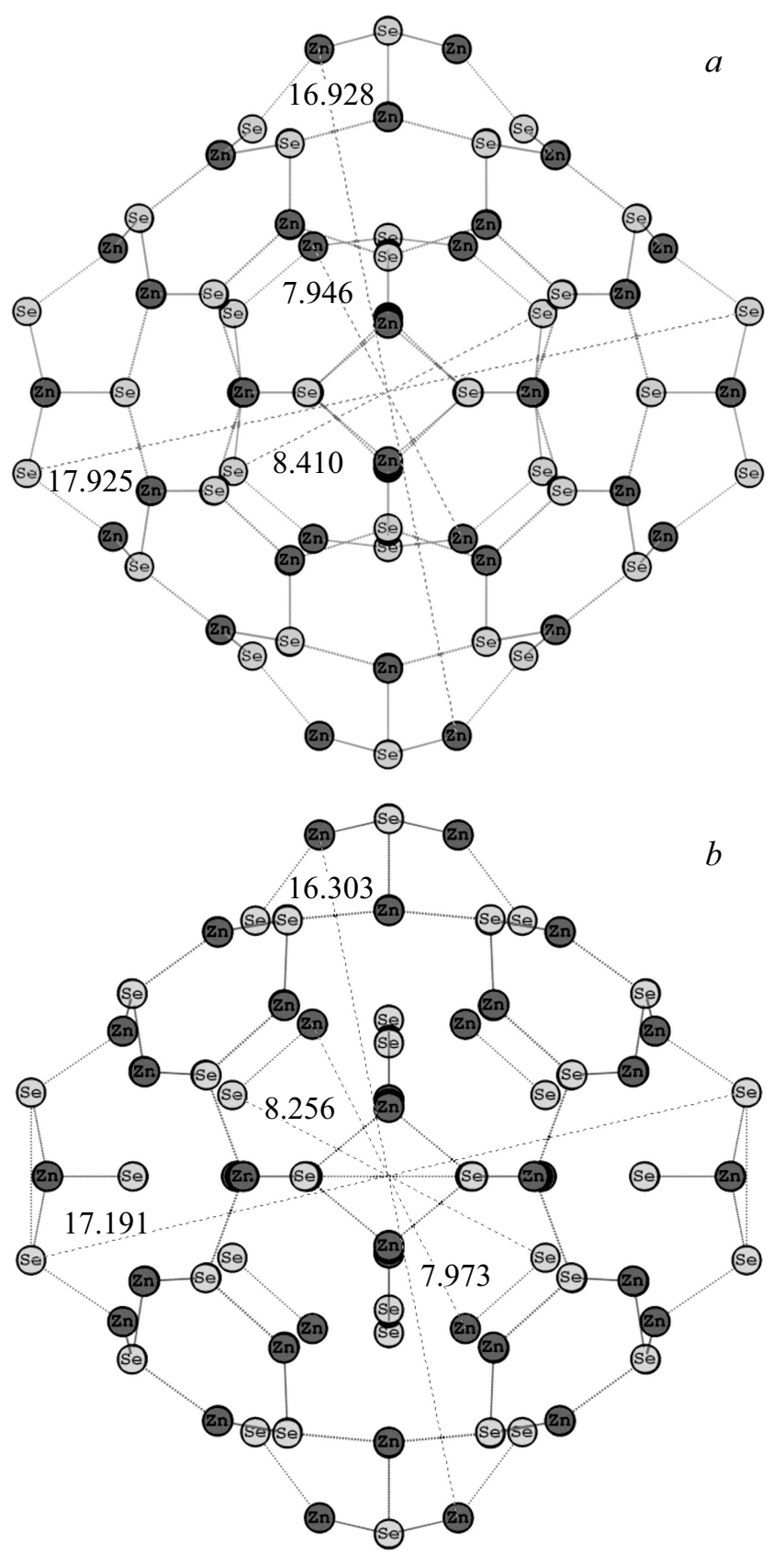

Рис. 3. Геометрия кластеров $\mathrm{ZnSe}$ после релаксации: (a) ониона $\mathrm{Zn}_{12} \mathrm{Se}_{12} @ \mathrm{Zn}_{48} \mathrm{Se}_{48} ;(b)$ кластера $\mathrm{Zn}_{60} \mathrm{Se}_{60}$ со смешанным $s p^{2} / s p^{3}$-типом связи. Размер между центрами атомов показан в $\AA$.

кольце исследованных кластеров $0.243-0.256 \mathrm{~nm}$ коррелирует с экспериментальными данными [22] длиной связи $0.2442 \mathrm{~nm}$ в твердотельном ZnSe структуры сфалерита при постоянной решетки $0.5668 \mathrm{~nm}$. Анализ исследования кластеров $\mathrm{Zn}_{n} \mathrm{Se}_{n}$ показал, что наиболее близок к твердотельному $\mathrm{ZnSe}$ кластер $\mathrm{Zn}_{48} \mathrm{Se}_{48}$, у которого длина связи $0.243-0.246 \mathrm{~nm}$ близка длине связи в твердотельном ZnSe структуры сфалерита $0.2442 \mathrm{~nm}$ и ширина энергетической щели $E_{g}=2.33 \mathrm{eV}$ при $E_{g}=2.82 \mathrm{eV}$ у твердотельного $\mathrm{ZnSe}$.
Для структур на основе $\mathrm{CdSe}$ наиболее стабильным оказался сферический $(\mathrm{CdSe})_{60}$ кластер со смешанными $s p^{2} / s p^{3}$-связями, у которого энергия когезии на пару атомов $\mathrm{Cd}-\mathrm{Se}$ равна $3.424 \mathrm{eV}$. Онион $\mathrm{Cd}_{12} \mathrm{Se}_{12} @ \mathrm{Cd}_{48} \mathrm{Se}_{48}$, близок к нему, его энергия когезии на пару атомов $\mathrm{Cd}-\mathrm{Se}$ равна $3.41 \mathrm{eV}$. Длина связи $\mathrm{Cd}-\mathrm{Se}$ в шестичленном кольце исследованных кластеров 0.262-0.277 коррелирует с экспериментальными данными [22] длиной связи в твердотельном CdSe структуры сфалерита $0.261 \mathrm{~nm}$ при постоянной решетки

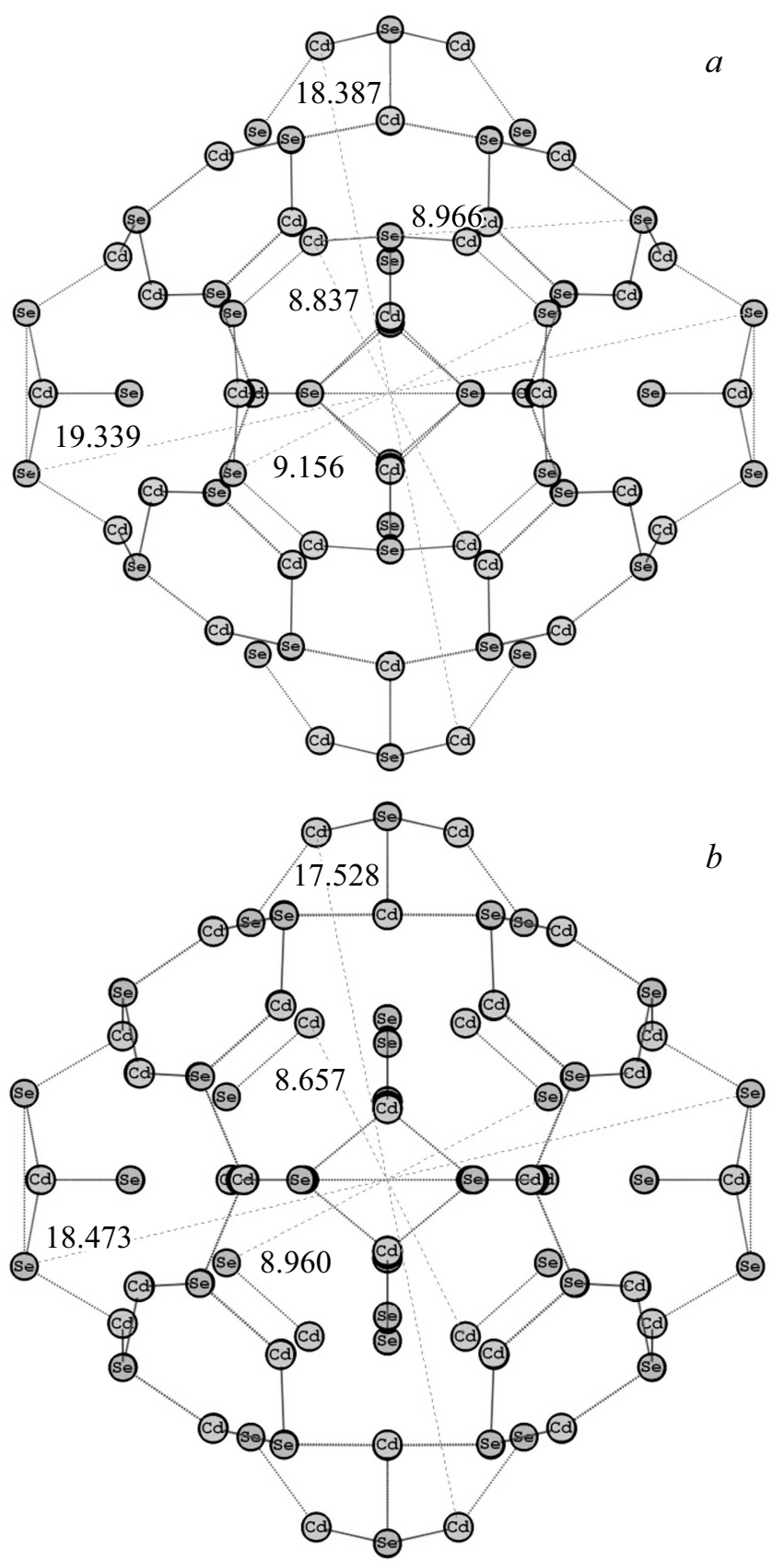

Pис. 4. Геометрия кластеров $\mathrm{CdSe}$ после релаксации: $(a)$ ониона $\mathrm{Cd}_{12} \mathrm{Se}_{12} @ \mathrm{Cd}_{48} \mathrm{Se}_{48} ;(b)$ кластера $\mathrm{Cd}_{60} \mathrm{Se}_{60}$ со смешанным $s p^{2} / s p^{3}$-типом связи. Размер между центрами атомов показан B $\AA$. 

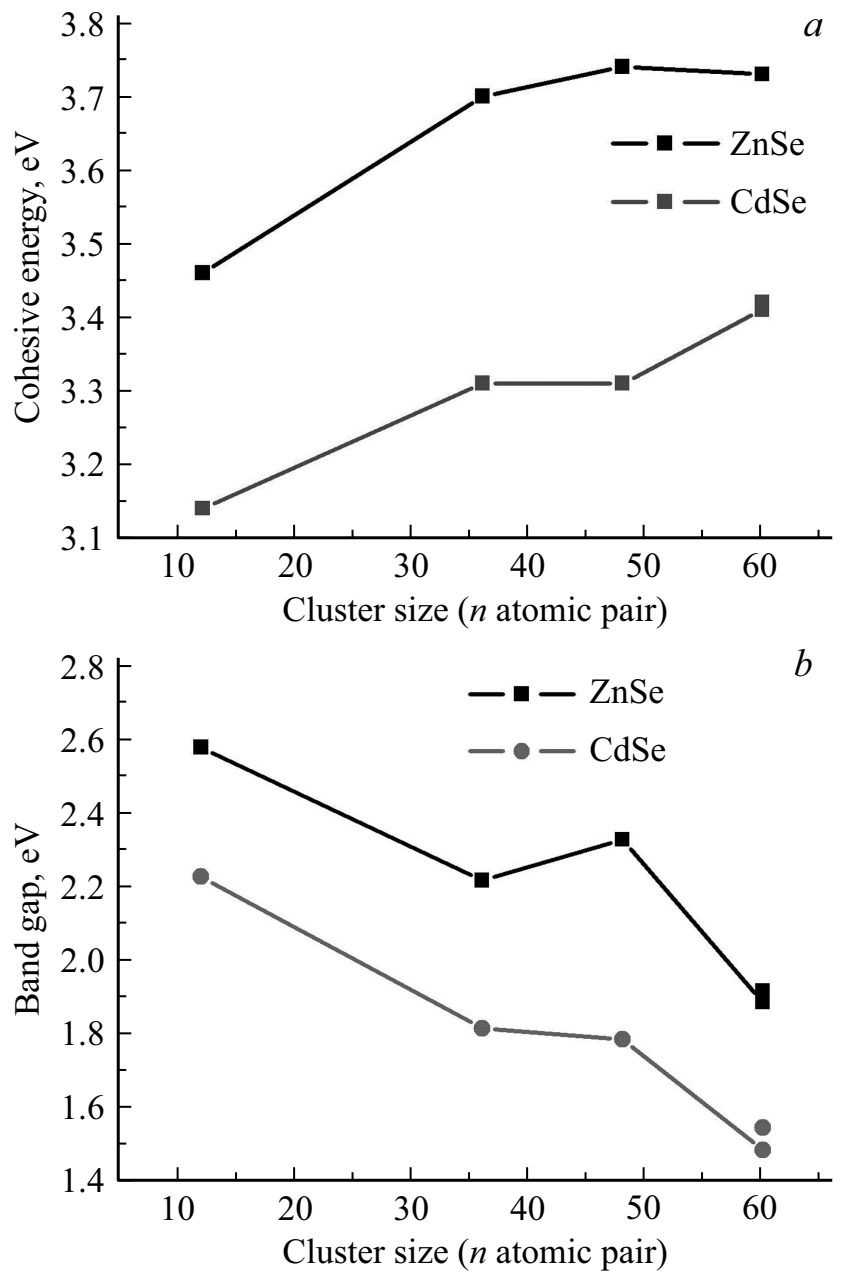

Рис. 5. Зависимость энергии когезии $(a)$ и ширины запрещенной щели $(b)$ кластеров $(\mathrm{ZnSe})_{n}$ и $(\mathrm{CdSe})_{n}(n=12,36,48$, $60)$ от размера кластера (числа $\mathrm{Zn}-\mathrm{Se}, \mathrm{Cd}-\mathrm{Se}$ атомных пар в кластере).

$0.6052 \mathrm{~nm}$. У кластера $\mathrm{Cd}_{48} \mathrm{Se}_{48}$ длина связи в пределах 0.2624-0.267 nm. Наиболее соответствует твердотельному $\mathrm{CdSe}$ кластер $\mathrm{Cd}_{48} \mathrm{Se}_{48}$, у которого длина связи $0.262-0.267 \mathrm{~nm}$ близка длине связи в твердотельном $\mathrm{CdSe}$ структуры сфалерита $0.261 \mathrm{~nm}$ и ширина энергетической щели $E_{g}=1.79 \mathrm{eV}$ при $E_{g}=1.74 \mathrm{eV}$ у твердотельного CdSe.

Анализ длины связи, размера кластера между центрами атомов и расстояния между поверхностным и внутренним слоями в онионе $\mathrm{Zn}_{12} \mathrm{Se}_{12} @ \mathrm{Zn}_{48} \mathrm{Se}_{48}$, сферическом $(\mathrm{ZnSe})_{60}$, а также онионе $\mathrm{Cd}_{12} \mathrm{Se}_{12} @ \mathrm{Cd}_{48} \mathrm{Se}_{48}$ и сферическом $(\mathrm{CdSe})_{60}$ представлен в табл. 1,2 и на рис. 3,4 .

В процессе поиска равновесной геометрии в онионе $\mathrm{Zn}_{12} \mathrm{Se}_{12} @ \mathrm{Zn}_{48} \mathrm{Se}_{48}$ длина связи несущественно увеличилась (с $0.2434-0.2466 \mathrm{~nm}$ до $0.249-0.2503 \mathrm{~nm}$ ), расстояние между слоями после релаксации составило $0.3195-0.3314 \mathrm{~nm}$. Таким образом, структура ониона сохранилась, что видно и на карте распределения электронной плотности (рис. 6). В кластере $(\mathrm{ZnSe})_{60}$ в процессе оптимизации геометрии длина связи во внутреннем и наружном слое практически не изменилась. Связи между амомами внутреннего и наружного слоя удлиняются, присходит почти расслоение кластера на два слоя. Расстояние между оболочками составило $0.2765-0.2968 \mathrm{~nm}$, что значительно больше длины связи в твердотельной структуре $(0.243 \mathrm{~nm})$, но меньше, чем расстояние между слоями в онионе $(0.33 \mathrm{~nm})$. Исследование расчета сферического $(\mathrm{ZnSe})_{60}$ кластера показало, что в процессе оптимизации связи между атомами внутреннего и наружного слоя удлиняются, присходит почти расслоение кластера на два слоя.

Аналогичный анализ для кластеров CdSe показал, что после оптимизации онион $\mathrm{Cd}_{12} \mathrm{Se}_{12} @ \mathrm{Cd}_{48} \mathrm{Se}_{48}$ сохраняет свою структуру, расстояние между слоями равно $0.318-0.336 \mathrm{~nm}$. В кластере $(\mathrm{CdSe})_{60}$ со смешанным типом связи $s p^{2} / s p^{3}$-расстояние между слоями после оптимизации геометрии увеличивается и становится равно $0.2876-0.2979 \mathrm{~nm}$, что значительно больше длины связи
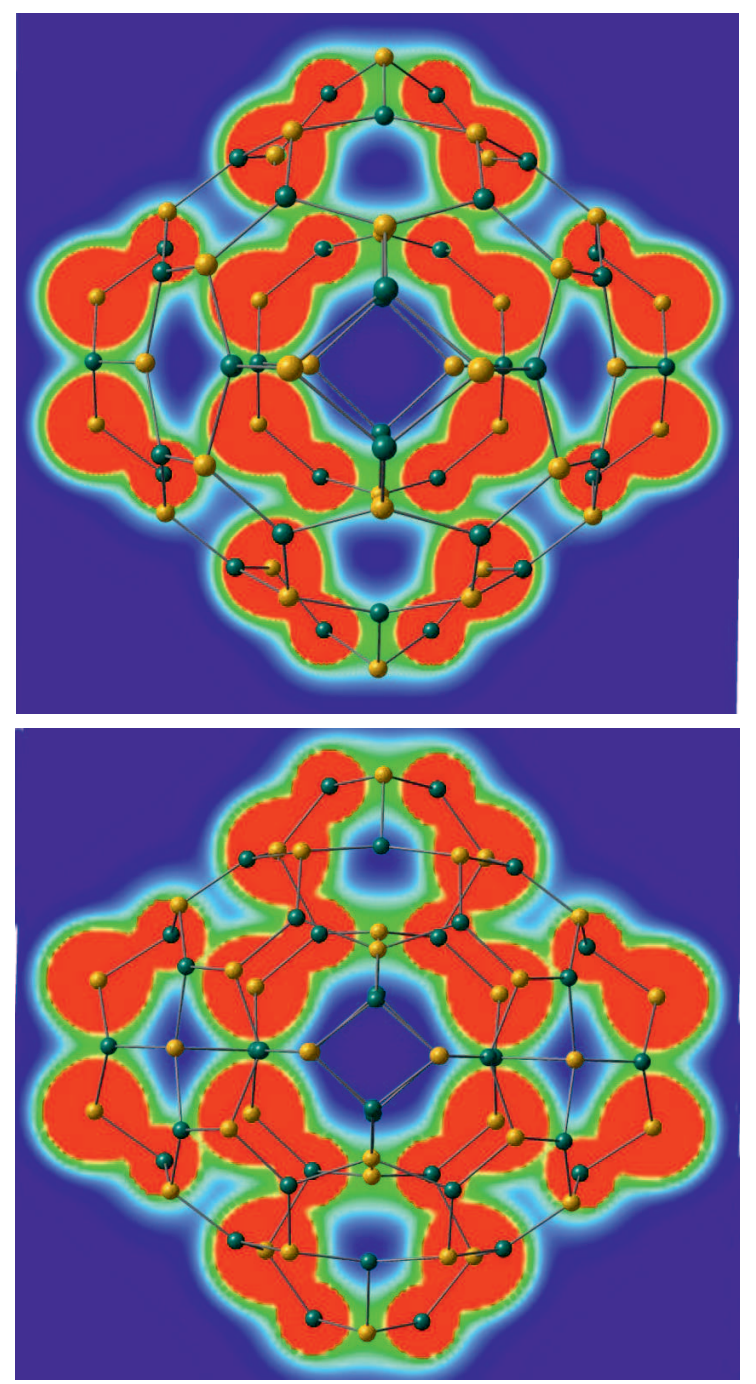

Рис. 6. Карты распределения электронной плотности, проходящие через центральную плоскость кластеров: $\mathrm{Zn}_{12} \mathrm{Se}_{12} @ \mathrm{Zn}_{48} \mathrm{Se}_{48}(a)$ и $\mathrm{Zn}_{60} \mathrm{Se}_{60}(b)$. 
Таблица 1. Энергетические и геометрические параметры кластеров $\mathrm{ZnSe}:\left(E_{c} /\right.$ pare-atom-энергия когезии на пару атомов $\mathrm{Zn}-\mathrm{Se}, \mathrm{eV} ; E_{g}$ - ширина энергетической щели, $\mathrm{eV}$; размер кластера - расстояние между центрами диаметрально расположенных атомов, nm; длина связи — расстояние между центрами атомов в шестичленных кольцах, $\mathrm{nm}$ )

\begin{tabular}{l|c|l|c|c}
\hline \multicolumn{1}{c|}{ Частица } & $\begin{array}{c}E_{c} / \text { pare-atom } \\
\mathrm{Zn}-\mathrm{Se}, \\
\mathrm{eV}\end{array}$ & $\begin{array}{c}E_{g}, \\
\mathrm{eV}\end{array}$ & $\begin{array}{c}\text { Размер } \\
\text { кластера, } \\
\mathrm{nm}\end{array}$ & $\begin{array}{c}\text { Длина } \\
\text { связи, } \\
\mathrm{nm}\end{array}$ \\
\hline $\mathrm{Zn}_{12} \mathrm{Se}_{12}$ & 3.456 & 2.58 & 0.835 & 0.247 \\
$\mathrm{Zn}_{36} \mathrm{Se}_{36}$ & 3.694 & 2.22 & 1.497 & 0.245 \\
$\mathrm{Zn}_{48} \mathrm{Se}_{48}$ & 3.739 & 2.329 & 1.81 & $0.243-0.246$ \\
$\mathrm{Zn}_{12} \mathrm{Se}_{12} @ \mathrm{Zn}_{48} \mathrm{Se}_{48}$ & 3.732 & 1.894 & 1.792 & $0.249-0.256$ \\
$(\mathrm{ZnSe})_{60}$ & 3.727 & 1.921 & 1.72 & $0.247-0.254$
\end{tabular}

Таблица 2. Энергетические и геометрические параметры кластеров CdSe: $\left(E_{c} /\right.$ pare-atom - энергия когезии на пару атомов $\mathrm{Cd}-\mathrm{Se}, \mathrm{eV} ; E_{g}$ - ширина энергетической щели, $\mathrm{eV}$; размер кластера - расстояние между центрами диаметрально расположенных атомов, nm; длина связи - расстояние между центрами атомов в шестичленных кольцах, $\mathrm{nm}$ )

\begin{tabular}{l|c|c|c|c}
\hline \multicolumn{1}{c|}{ Частица } & $\begin{array}{c}E_{c} / \text { pare-atom } \\
\mathrm{Cd}-\mathrm{Se}, \\
\mathrm{eV}\end{array}$ & $\begin{array}{c}E_{g}, \\
\mathrm{eV}\end{array}$ & $\begin{array}{c}\text { Размер } \\
\text { кластера, } \\
\mathrm{nm}\end{array}$ & $\begin{array}{c}\text { Длина } \\
\text { связи, } \\
\mathrm{nm}\end{array}$ \\
\hline $\mathrm{Cd}_{12} \mathrm{Se}_{12}$ & 3.142 & 2.226 & 0.899 & 0.268 \\
$\mathrm{Cd}_{36} \mathrm{Se}_{36}$ & 3.311 & 1.815 & 1.64 & $0.268-0.271$ \\
$\mathrm{Cd}_{48} \mathrm{Se}_{48}$ & 3.307 & 1.793 & 1.97 & $0.262-0.267$ \\
$\mathrm{Cd}_{12} \mathrm{Se}_{12} @ \mathrm{Cd}_{48} \mathrm{Se}_{48}$ & 3.41 & 1.491 & 1.934 & $0.27-0.279$ \\
$(\mathrm{CdSe})_{60}$ & 3.424 & 1.546 & 1.847 & $0.269-0.277$
\end{tabular}

в твердотельной структуре $(0.2624 \mathrm{~nm})$, но меньше, чем расстояние между слоями в онионе $(0.336 \mathrm{~nm})$.

Для анализа полученных результатов проведено сравнение с результатами первопринципных расчетов атомной и электронной структуры пленки на основе $\mathrm{ZnSe}$ и $\mathrm{CdSe}$ со структурой сфалерита [23]. В [23] показано, что в процессе оптимизации геометрии структуры изначальная гофрировка верхнего слоя пленки уменьшается, а расстояние между поверхностным и нижележащим слоями увеличивается, релаксация приводит к расщеплению атомных слоев. Расстояние между слоями пленки структуры сфалерита составило 0.248 и $0.267 \mathrm{~nm}$ для $\mathrm{ZnSe}$ и CdSe соответственно.

Для оптимизированной геометрии кластеров $(\mathrm{ZnSe})_{60}$ и $(\mathrm{CdSe})_{60}$ расстояние между поверхностным и внутренним слоями составило $0.276-0.297 \mathrm{~nm}$ и $0.288-0.298 \mathrm{~nm}$ для кластеров $(\mathrm{ZnSe})_{60}$ и $(\mathrm{CdSe})_{60}$ соответственно.

Таким образом, расчет атомной структуры пленки структуры сфалерита для $\mathrm{ZnSe}$ и $\mathrm{CdSe}$ коррелирует с результатом полученным для изолированных кластеров $(\mathrm{ZnSe})_{60}$ и $(\mathrm{CdSe})_{60}$.

\section{4. Заключение}

Анализ исследования кластеров $\mathrm{Zn}_{n} \mathrm{Se}_{n}$ показал, что наиболее соответствует твердотельному $\mathrm{ZnSe}$ кластер $\mathrm{Zn}_{48} \mathrm{Se}_{48}$, у которого длина связи близка длине связи в твердотельном $\mathrm{ZnSe}$ структуры сфалерита и ширина энергетической щели близка ширине запрещенной зоны у твердотельного $\mathrm{ZnSe}$.

Анализ исследования кластеров $\mathrm{Cd}_{n} \mathrm{Se}_{n}$ показал, что наиболее соответствует твердотельному CdSe кластер $\mathrm{Cd}_{48} \mathrm{Se}_{48}$, у которого длина связи близка длине связи в твердотельном CdSe структуры сфалерита и ширина энергетической щели близка ширине запрещенной зоны у твердотельного CdSe.

В процессе оптимизации геометрии сферичесих $(\mathrm{ZnSe})_{60}$ и $(\mathrm{CdSe})_{60}$ кластеров показано, что связи между атомами внутреннего и поверхностного слоя удлиняются, присходит отщепление верхнего слоя.

$\mathrm{B}$ качестве базовой модели для дальнейших исследований влияния примесей, собственных дефектов и нарушения стехиометрии изолированных кластеров и КТ на основе ZnSe и CdSe могут применяться ФП-кластеры $\mathrm{Zn}_{n} \mathrm{Se}_{n}$ и $\mathrm{Cd}_{n} \mathrm{Se}_{n}$.

\section{Список литературы}

[1] I. Tiginyanu, P. Topala, V. Ursaki. Nanostructures and Thin Films for Multifunctional Applications. Springer (2016). $576 \mathrm{c}$.

[2] A.I. Ekimov, A.A. Onushchenko. JETP Lett. 34, 345 (1981).

[3] Д.А. Куршанов, В.А. Осипова, И.В. Мартыненко. Оптические свойства квантовых точек $\mathrm{CdSe} / \mathrm{CdS}$ с различной толщиной оболочки. Сб. тез. докл. конгресca молодых ученых. http://kmu.ifmo.ru/collections_article/ 5252/opticheskie_svoystva_kvantovyh_tochek_CdSe/CdS_s_ razlichnoy_tolschinoy_obolochki.htm

[4] Р.Б. Васильев, В.С. Виноградов, С.Г. Дорофеев, С.П. Козырев, И.В. Кучеренко, Н.Н. Новикова. ФТТ 49, 523 (2007).

[5] А.А. Ващенко, А.Г. Витухновский, В.С. Лебедев, А.С. Селюков, Р.Б. Васильев, М.С. Соколикова. Письма в ЖЭТФ 100, 94 (2014).

[6] Р.Б. Васильев, М.С. Соколикова, А.Г. Витухновский, С.А. Амброзевич, А.С. Селюков, В.С. Лебедев. Квант. электроника 45, 9, 853 (2015).

[7] Jiwoong Yang, Franziska Muckel, Woonhyuk Baek, Rachel Fainblat, Hogeun Chang,Gerd Bacher, Taeghwan Hyeon. Chemical Synthesis, Doping, and Transformation of MagicSized Semiconductor Alloy Nanoclusters. Am. Chem. Soc. (2017).

https://www.researchgate.net/publication/316803325

[8] Biplab Goswami, Sougata Pal, Pranab Sarkar, G. Seifert, M. Springborg. Phys. Rev. B 73, 205312-1 (2006).

[9] L. Ovsiannikova, V. Kartuzov, I. Shtepliuk, G. Lashkarev. Acta Phys. Polonica A 129, A-41 (2016).

[10] I. Shtepliuk, V. Khranovskyy, G. Lashkarev, V. Khomyak, V. Lazorenko, A. Ievtushenko, M. Syväjärvi, V. Jokubavicius, R. Yakimova. Solid State Electron. 81, 72 (2013).

[11] L. Ovsiannikova, M. Dranchuk, G. Lashkarev, V. Kartuzov, M. Godlewski. Superlatt. Microstructur. 107, 1 (2017). 
[12] V.V. Pokropivny, L.I. Ovsyannikova. Phys. Solid State 49, 535 (2007).

[13] Z. Zhu, A. Chutia, R. Sahnoun, M. Koyama, H. Tsuboi, N. Hatakeyama, A. Endou, H. Takaba, M. Kubo, C.A. DelCarpio, A. Miyamoto. Jpn J. Appl. Phys. 47, 2999 (2008).

[14] Amit Jain, Vijay Kumar, Yoshiyuki Kawazoe. Comput. Mater. Sci. 36, 258 (2006).

[15] V.V. Pokropivny, V.V. Skorokhod, G.S. Oleinik, A.V. Kurdyumov, T.S. Bartnitskaya, A.V. Pokropivny, A.G. Sisonyuk, D.V. Sheichenko. J. Solid State Chem. 154, 214 (2000).

[16] L.I. Ovsyannikova, V.V. Pokropivny, V.L. Bekenev. Phys. Solid State 51, 2199 (2009).

[17] В.Я. Шевченко, А.Е. Мадисон. Физика и химия стекла 32 , 1, 118 (2006).

[18] А.Н. Еняшин, А.Л. Ивановский. ФТТ 49, 378 (2007).

[19] S. Huzinaga, J. Andzelm, M. Klobukowski, E. RadzioAndzelm, Y. Sakai, H. Tatewaki. Gaussian Basis Sets for Molecular Calculations. Elsevier, Amsterdam (1984).

[20] M.W. Schmidt, K.K. Baldridge, J.A. Boatz, S.T. Elbert, M.S. Gordon, J.H. Jensen, S. Koseki, N. Matsunaga, K.A. Nguyen, S.J. Su, T.L. Windus, M.Dupuis, J.A. Montgomery. J. Comput. Chem. 14, 1347 (1993).

[21] S. Portmann. Chimia 54, 766 (2000).

[22] Landolt-Bornstein / Ed. O. Madelung. New Ser. III. 22. Springer, Berlin (1987).

[23] В.Л. Бекенев, С.М. Зубкова. ФТТ 60, 187 (2018).

Редактор Т.Н. Василевская 